\title{
Syndrome of Supine Hypertension with Orthostatic Hypotension. A Nightmare for Physicians
}

\author{
AAMIR AHMED, BS ${ }^{1}$, BLAIR GRUBB, MD, FACC ${ }^{2}$, LIAQAT ZAMAN, MD, FACC, FHRS ${ }^{1}$ and \\ KHALIL KANJWAL, MD, FACC, FHRS, CCDS ${ }^{1}$ \\ ${ }^{1}$ Michigan Cardiovascular Institute, Central Michigan University, Saginaw, MI \\ ${ }^{2}$ Division of Cardiology, Department of Medicine, The University of Toledo Medical Center, $\mathrm{OH}$
}

\begin{abstract}
Supine hypertension-orthostatic hypotension $(\mathrm{SH} / \mathrm{OH})$ is a form of autonomic dysfunction characterized by hypertension when patients are supine and a clinically significant drop in blood pressure when they assume an upright posture. Treatment of this group of patients can be very challenging. In this review we attempt to outline the pathophysiology of this condition as well as potential management strategies for these patients.
\end{abstract}

KEYWORDS. concurrent supine hypertension, orthostatic hypotension.
ISSN 2156-3977 (print) ISSN 2156-3993 (online)

(C) 2016 Innovations in Cardiac Rhythm Management

\section{Introduction}

Orthostatic hypotension $(\mathrm{OH})$ is a condition that is relatively common in elderly patients and those who suffer from diabetes mellitus and Parkinson's disease. Some patients with $\mathrm{OH}$ will also develop concurrent supine hypertension $(\mathrm{SH})$. This results from a defect in the functioning of the baroreceptors that normally maintain appropriate blood pressure upon assuming an upright posture.

The goal of this paper to is to review the current data on $\mathrm{OH}$ with concurrent $\mathrm{SH}(\mathrm{SH} / \mathrm{OH})$ in an attempt to better characterize patterns of disease progression, risk factors for development of the syndrome, as well as potential treatments. It is our hope that this review will help physicians involved in the care of these patients to better understand this challenging syndrome and improve the quality of patient care.

The authors report no conflicts of interest for the published content. Manuscript received January 4, 2016, final version accepted March 2, 2016.

Address correspondence to: Khalil Kanjwal, MD, FACC, FHRS, CCDS, Assistant Professor of Medicine, Central Michigan University, Staff Electrophysiologist, Michigan Cardiovascular Institute, 1015 S. Washington Avenue, Saginaw MI. 48601. E-mail: khalilkanjwal@ yahoo.com

\section{Normal physiology}

The physiology of standing is complex. Upon assuming an upright posture, up to $800 \mathrm{ml}$ of blood is displaced downward, resulting in a relative state of central hypovolemia. This results in a significant drop in venous return and subsequently in the cardiac output as well. ${ }^{1}$ However, the baroreceptors normally detect this transient drop in venous return and respond by augmenting sympathetic outflow and inhibiting parasympathetic release. The net result is an increase in vascular tone and heart rate (and myocardial contractility) to help compensate for pooling of the blood in lower extremity venous system, which in turn helps to maintain the blood pressure at a near constant level. $^{2}$

The body must actively and quickly compensate for this relative hypovolemia to avoid a state of cerebral hypoperfusion. The primary mechanism by which the body adjusts to the drop in central blood pressure relies on increasing peripheral vascular resistance in response to signals received by arterial baroreceptors throughout the body, especially those in the carotid sinus. In addition to the effects of the baroreceptors, stretch receptors also play an important role in adjusting to an upright posture. Decreased firing from central stretch receptors (receptor unloading) indirectly leads to an increase in sympathetic outflow thus resulting in an increase in systemic vasoconstriction. ${ }^{1,2}$ 
These neurohumoral effects on vascular tone are further amplified by hormonal changes. These include stimulation of the renin-angiotensin-aldosterone axis and increased release of vasopressin. Both effects serve to increase blood pressure and maintain normal cerebral perfusion upon assuming the upright posture. ${ }^{3}$

\section{Pathophysiology}

Pathophysiology of orthostatic hypotension. $\mathrm{OH}$ is defined as a reduction in systolic blood pressure by at least $20 \mathrm{mmHg}$ or reduction in diastolic blood pressure by at least $10 \mathrm{mmHg}$ within $3 \mathrm{~min}$ of assuming an upright posture. ${ }^{1}$ This condition commonly affects patients suffering from diabetes mellitus and Parkinson's disease. Etiologies of $\mathrm{OH}$ include neurogenic causes (primary and secondary dysautonomia), relative hypovolemia, and pharmacological agents. ${ }^{2}$

As noted earlier, standing promotes the pooling of around $800 \mathrm{ml}$ of blood to the lower extremities and other dependent body compartments, which reduces venous return, cardiac output, and blood pressure. Normally, this should initiate a compensatory reflex mediated by baroreceptors in the carotid sinus and aortic arch. This reflex serves to increase cardiac output and prevent the fall in blood pressure caused by switching to an upright posture. This is accomplished by increasing sympathetic outflow and decreasing parasympathetic outflow. When this reflex fails, cerebral hypoperfusion can occur, leading to symptoms of orthostatic intolerance such as dizziness, lightheadedness, presyncope, and syncope. ${ }^{3}$

Normally, standing upright leads to decreased firing from glossopharyngeal and vagal afferent baroreceptors (in the carotid sinus and aortic arch). This decreased firing is sensed by the nucleus of the solitary tract (NTS) in the dorsomedial medulla. The NTS then transmits this signal of reduced baroreceptor firing to the nucleus ambiguus, which then reduces vagal/parasympathetic input to the entire heart, including both the sinoatrial and atrioventricular nodes. Sympathetic activity is increased when the NTS projects to the caudal ventrolateral medulla, which then connects to the rostral ventrolateral medulla, leading to activation of sympathetic ganglia. Any abnormalities in these pathways can result in deficient sympathetic activation or parasympathetic inhibition, potentially leading to decreased cerebral blood flow and orthostatic hypotension. ${ }^{1-3}$

What is supine hypertension? There is no formal definition for SH. However, it has been proposed that $\mathrm{SH}$ might be considered as a systolic blood pressure of at least $150 \mathrm{mmHg}$ and a diastolic blood pressure of at least $90 \mathrm{mmHg}$ when supine. ${ }^{4-7}$

Pathophysiology of SH/OH. SH/OH develops in many patients with $\mathrm{OH}$ and autonomic failure, which describes a condition where sympathetic outflow is inhibited because postganglionic neurons are deficient in releasing norepinephrine, especially when upright. $\mathrm{OH}$ would develop, as the body is unable to adjust appropriately to the upright posture by inducing an adequate sympathetic response. ${ }^{4}$
This could result in $\mathrm{SH}$, and, possibly because of deficient baroreflex function, increased volume of blood, improper natriuresis, and residual sympathetic output in the setting of hypersensitive postsynaptic adrenergic receptors. In this way, autonomic failure is a significant risk factor for $\mathrm{SH} / \mathrm{OH}^{5}$ Long-term hypertension leads to desensitization of the baroreceptor reflex, which might also contribute to the pathogenesis of $\mathrm{SH} / \mathrm{OH}$. Another contributing factor to $\mathrm{SH} /$ $\mathrm{OH}$ could be residual sympathetic tone acting on postsynaptic adrenergic receptors that have become hypersensitive. This mechanism suggests that patients with chronic hypertension may be at risk for developing $\mathrm{OH}$ because of altered sensitivity of both baroreceptors and adrenergic receptors especially when assuming an upright posture., Patients with $\mathrm{OH}$ because of autonomic failure (primary or secondary) may at times develop supine hypertension, which could be a result of the medications used for the treatment of $\mathrm{OH}$. It may also be a result of baroreflex dysfunction in the presence of residual sympathetic outflow, particularly in patients with central autonomic degeneration. $^{3}$

\section{Associated conditions of $\mathrm{SH} / \mathrm{OH}$}

$\mathrm{SH} / \mathrm{OH}$ in Parkinson's: an example of autonomic failure causing $\mathrm{SH} / \mathrm{OH}$. $\mathrm{SH} / \mathrm{OH}$ has often been observed in Parkinson's disease. $\mathrm{OH}$ itself occurs in about $40 \%$ of patients with Parkinson's disease and most often as a result of nervous system dysfunction. ${ }^{5}$ Sharabi and Goldstein examined patients with $\mathrm{OH}$ and Parkinson's and revealed that these patients (" $\mathrm{PD}+\mathrm{OH}^{\prime \prime}$ ) exhibited baroreflex sympathoneural and cardiovagal failure. ${ }^{5}$ These patients were also found to have significant "sympathetic noradrenergic denervation" especially in the left ventricular myocardium. ${ }^{8}$ According to Sharabi and Goldstein, these patients also have decreased levels of norepinephrine in their plasma compared with patients with Parkinson's without $\mathrm{OH}^{8}$ This baroreflex instability coupled with decreased sympathetic innervation throughout the body could explain the development of $\mathrm{OH}$ in these patients.

$\mathrm{SH}$ in Parkinson's patients with $\mathrm{OH}$ usually occurs during the night; however, the mechanism of SH in PD is not clearly known. ${ }^{8}$ The mechanism of $\mathrm{SH}$ and $\mathrm{OH}$ could very well share a common mechanism related to the baroreflex failure. $\mathrm{SH}$ during the night might evoke pressure related natriuresis, which can result in relative daytime hypovolemia and $\mathrm{OH}^{8}$

Drugs causing $\mathrm{SH} / \mathrm{OH}$. Antihypertensive medications used to treat $\mathrm{SH}$ and sympathomimetic drugs to treat $\mathrm{OH}$ may result in an $\mathrm{SH} / \mathrm{OH}$ syndrome. Inappropriate timing of drug dosage might worsen this effect. As a result, short-acting antihypertensive should be employed to treat hypertension in these patients to avoid exacerbating $\mathrm{OH}$. Likewise, adrenergic agents to treat $\mathrm{OH}$ should be timed appropriately in order to avoid nighttime $\mathrm{SH}^{8}$

\section{Associated symptoms}

Patients with $\mathrm{SH} / \mathrm{OH}$ may be asymptomatic, but symptoms of orthostatic intolerance such as "dizziness, lightheadedness, and/or loss or near-loss of consciousness" 
may be present. ${ }^{6}$ Patients with $\mathrm{SH} / \mathrm{OH}$ exhibited lower cognitive performance than those patients with $\mathrm{SH}$ without $\mathrm{OH}$. The pathophysiology of this finding is potentially related to increased cerebral arteriosclerosis and white matter damage. These are both associated with cerebral hypoperfusion and lower cognitive performance. ${ }^{9,10}$ Jones et al. ${ }^{11}$ have explored the importance of $\mathrm{OH}$ as a predictor for the development of heart failure, especially in those around the age of 50 years.

\section{Treatment options/management}

The treatment of $\mathrm{SH} / \mathrm{OH}$ can be very challenging for the physician. This is partly because the etiology of the syndrome can be difficult to delineate. Additionally, patients can have very different responses to standard therapy, so outcomes can vary greatly from case to case. Naschitz et $\mathrm{al}^{5}$ described the variability of $\mathrm{SH} / \mathrm{OH}$ presentations and proposes a classification system for $\mathrm{SH} / \mathrm{OH}$ syndrome, characterized by clinical presentation (e.g. acute or chronic, typical, or atypical), pathophysiology (e.g. autonomic dysfunction, organ failure, drug effects), and hemodynamic patterns on autonomic function tests such as the head-up tilt test. ${ }^{2}$ Treatment of $\mathrm{OH}$ with $\mathrm{SH}$ is further complicated by the fact that treating one aspect of the condition may exacerbate the other.

Sharabi and Goldstein ${ }^{8}$ have suggested that $\mathrm{OH}$ and $\mathrm{SH}$ should be treated separately, and that non-pharmacological interventions should be employed first. For $\mathrm{OH}$, these include smaller, more frequent meals and increasing water intake, along with other physical maneuvers to increase blood pressure (such as squatting). As $\mathrm{SH}$ or $\mathrm{OH}$ becomes more severe, pharmacological measures should be taken, such as adrenergic agonists for $\mathrm{OH}$ or short-acting antihypertensives for $\mathrm{SH}$. These medications must be strictly monitored to minimize any undesired side effects that may worsen $\mathrm{SH}$ or $\mathrm{OH} .{ }^{5}$ They strongly recommend the use of 24-hour blood pressure monitoring as a means to identify the timing and magnitude of daily fluctuations. This practice can further help to guide treatment. ${ }^{8}$

Lamarre-Cliché $^{7}$ shed more light on treatment options for patients with $\mathrm{SH} / \mathrm{OH}$. This paper asserts that treatment of $\mathrm{SH} / \mathrm{OH}$ should begin with a discussion with the patient of the therapeutic goals of treatment. It is difficult to treat all symptoms of the disease; so understanding the patient's mindset is crucial to improving their quality of life. This paper states that treatment of $\mathrm{OH}$ is highly focused on symptoms that can have significant effects on quality of life, whereas treatments of $\mathrm{SH}$ are largely focused on preventing hypertension-induced organ damage. ${ }^{7}$
In our practice, we first carefully review the medications the patient is currently taking and attempt to reduce or eliminate any unnecessary agents which may be contributing to the patients condition. Oftentimes older patients are on a multitude of medications that potentially interact with one another and interfere with the physiologic mechanisms that help regulate orthostatic control. Some common medications have been associated with an increased risk of falls in older patients (such as sedatives, hypnotics, antidepressants, and benzodiazepines), which can further complicate management. We encourage patients to sit at the side of their beds for several minutes before attempting to stand. We also encourage them to sleep with the head of their beds elevated (often by placing a brick under each of the back bead-posts) or in a reclining chair. It is important for both physician and patient to realize that the goal of therapy is not to obtain perfect control of blood pressure; rather it is to maintain the blood pressure within an acceptable range that minimizes symptoms while at the same time preventing harm. This is especially true in patients with dramatic swings in blood pressure that occur when going from the supine to upright positions. In some ways these patients are similar to those with "brittle" insulindependent diabetes mellitus, in that with these patients too rigorous an attempt at controlling blood sugar may result in frequent and severe hypoglycemic events. In these diabetic patients somewhat higher than normal fasting blood sugars are often tolerated as a way of preventing serious hypoglycemic events. In an analogous manner we will tolerate supine blood pressures as high as $160-170 \mathrm{mmHg}$ in occasional patients if this proves effective in mitigating severe symptoms and preventing potentially disastrous falls from occurring.

To control extreme supine hypertension we often employ alpha-2 agonists such as clonidine, angiotensin receptor blockers, and combined alpha/beta blockers such as labetalol (Table 1). The drug pyridostigmine is often quite helpful in preventing supine $\mathrm{OH}$ without worsening $\mathrm{SH}$. In some $\mathrm{SH} / \mathrm{OH}$ patients the swings in blood pressure are so extreme that midodrine or droxidopa is administered when upright to prevent $\mathrm{OH}$. Clonidine $0.1 \mathrm{mg}$ is then given if severe hypertension develops. Preventing any degree of $\mathrm{OH}$ is often not a realistic goal. Instead, the aim is to maintain blood pressure at a level above the individual's threshold for maintenance of adequate cerebral perfusion. Discussing realistic goals for therapy with patients should emphasize the point that perfect control of blood pressure is unlikely, instead the aim is to maintain blood pressure within a safe range that

Table 1: Medications used in supine hypertension-orthostatic hypotension.

\begin{tabular}{llcl}
\hline Medication & \multicolumn{1}{c}{ Dosage } & Indication & Problems \\
\hline Clonidine & Tablet $0.1 \mathrm{mg}$ & $\mathrm{SH}$ & Dry mouth, patch 0.1 mg/weekly \\
Losartan & $25-100 \mathrm{mg}$ & $\mathrm{SH}$ & Edema \\
Labetalol & $50-200 \mathrm{mg}$ & $\mathrm{SH}$ & Fatigue \\
Pyridostigmine & $60 \mathrm{mg}$ orally three times daily & $\mathrm{OH}$ & Nausea/diarrhea \\
Midodrine & $5-10 \mathrm{mg}$ orally three times daily & $\mathrm{OH}$ & Nausea/headache \\
\hline
\end{tabular}

$\mathrm{OH}$ : orthostatic hypotension; $\mathrm{SH}$ : supine hypertension. 
ensures their safety and prevention of serious symptoms. Discussing realistic goals for therapy with patients using a multidisciplinary approach is important in ensuring their expectations are within reason. Providing a holistic perspective of the disease and its treatment can ultimately help patients cope with their condition and could potentially improve their quality of life significantly. ${ }^{10}$

\section{Latest research and future management of $\mathrm{SH} / \mathrm{OH}$}

As stated earlier, the treatment of $\mathrm{OH}$ should be aimed more at improving quality of life and prevention of injury, and the treatment of $\mathrm{SH}$ is primarily aimed at decreasing organ damage. ${ }^{4}$ However, the importance of $\mathrm{SH}$ in autonomic failure is still unclear. In addition, $\mathrm{SH}$ has been linked to left ventricular hypertrophy (LVH) and kidney dysfunction. ${ }^{12}$

There is still much we do not understand about the syndrome of $\mathrm{SH} / \mathrm{OH}$. Further research should help us secure a better appreciation of this condition while at the same time elaborating better therapeutic modalities.

\section{References}

1. Wieling W, VanLieshout JJ. Maintenance of postural normotension in humans. In: Low $\mathrm{P}$, ed. Clinical Autonomic Disorders. Philadelphia PA: Lippincott-Williams and Wilkins; 2008:57-67.

2. Thompson WO, Thompson PK, Dailey ME. The effect of posture on the composition and volume of the blood in man. J Clin Invest. 1928;5(4):573-604.
3. Benarroch E, Lipp A. Neural control of blood vessels. In: Low P, Benarroch E (eds). Clinical Autonomic Disorders. Philadelphia, PA: Lippincott-Williams and Wilkins; 2008:106-110.

4. Kanjwal K, George A, Figueredo VM, Grubb BP. Orthostatic hypotension: definition, diagnosis and management. J Cardiovasc Med (Hagerstown). 2015;16(2):75-81.

5. Naschitz JE, Slobodin G, Elias N, Rosner I. The patient with supine hypertension and orthostatic hypotension: a clinical dilemma. Postgrad Med J. 2006;82(966):246-253.

6. Longo DL, Kasper DL, Jameson JL, Fauci AS, Hauser SL, Loscalzo J. Syncope. In: Longo, D, ed. Harrison's Principles of Internal Medicine. 918th ed. New York, NY: The McGrawHill Companies, Inc; 2012:171-177.

7. Lamarre-Cliche M. Orthostatic hypotension and supine hypertension in the patient with autonomic failure. Can J Gen Intern Med. 2014;9(3):91-95.

8. Sharabi Y, Goldstein DS. Mechanisms of orthostatic hypotension and supine hypertension in Parkinson disease. J Neurol Sci. 2011;310(1-2):123-128.

9. Romero-Ortuno R, O'Connell MDL, Finucane C, Soraghan C, Fan CW, Kenny RA. Insights into the clinical management of the syndrome of supine hypertension - orthostatic hypotension (SH-OH): The Irish Longitudinal Study on Ageing (TILDA). BMC Geriatr. 2013;13:73.

10. Frewen J, Finucane C, Savva GM, Boyle G, Kenny RA. Orthostatic hypotension is associated with lower cognitive performance in adults aged 50 plus with supine hypertension. J Gerontol A Biol Sci Med Sci. 2014;69(7):878-885.

11. Jones CD, Loehr L, Franceschini N, et al. Orthostatic hypotension as a risk factor for incident heart failure: The atherosclerosis risk in communities study. Hypertension. 2012;59(5):913-918.

12. Garland EM, Gamboa A, Okamoto L, et al. Renal impairment of pure autonomic failure. Hypertension. 2009;54(5):1057-1061. 